\title{
Procesos de separación de las tierras raras ${ }^{(\bullet)}$
}

\author{
F.J. Alguacil ${ }^{(*)}$ y F. Rodríguez ${ }^{(* *)}$ \\ Resumen Los elementos pertenecientes al grupo de las tierras raras presentan un comportamiento peculiar, \\ consistente en que sus propiedades parecidas hacen que su separación con los procedimientos con- \\ vencionales no sea fácil. Este hecho, unido a las condiciones de alta pureza que su uso práctico \\ demanda, han llevado al desarrollo de nuevas tecnologías que permiten la separación y obtención de \\ estos elementos en condiciones de pureza elevada. En el presente trabajo se revisan algunos de los \\ resultados obtenidos con la aplicación se estas tecnologías en la separación y purificación de las tie- \\ rras raras.
}

Palabras clave: Tierras raras. Procesos de separación.

\section{Separation processes in rare earths}

\begin{abstract}
The rare earth elements are unique in its behaviour, its similar properties make very difficult its separation by conventional procedures, this linked to the necessity to obtain high grade final products, as demanded by modern industry, had led to the develop of new technologies that allow the final goal of the utilization of these rare elements: the separation and obtention of products which cope the demands of its uses are needed for. The present work revised some of the applications of these technologies in the separation and purification of rare earths.
\end{abstract}

Keywords: Rare earths. Separation processes.

\section{INTRODUCCIÓN}

$\mathrm{Al}$ grupo de las tierras raras pertenecen una serie de elementos que son muy difíciles de separar mediante las técnicas tradicionales, ya que poseen propiedades químicas muy parecidas.

Estas primeras técnicas utilizaban recristalizaciones múltiples y fraccionadas, operaciones que necesitaban la recirculación de muchas corrientes hasta obtener productos con una pureza adecuada.

La mayor demanda de estos elementos metálicos en la industria moderna, especialmente para diversas tecnologías avanzadas, ha necesitado la obtención de estos metales en un estado muy puro, imposible de alcanzar con las técnicas convencionales, por lo que se ha llevado a cabo un esfuerzo investigador y de desarrollo importante en los distintos campos sobre obtención y recuperación de las tierras raras de los diferentes recursos minerales que las contienen (como la monacita, bastaensita, xenotima y euxenita), e incluso, sobre el procesado de

$(\bullet)$ Trabajo recibido el día 19 de noviembre de 1996.

(*) Centro Nacional de Investigaciones Metalúrgicas (CSIC). Avda. de Gregorio del Amo, 8. 28040-Madrid (España).

(**) Área de Ciencia de Materiales e Ingeniería Metalúrgica. Universidad de Salamanca. Salamanca (España). otros metales de los que es posible obtener estos elementos como productos secundarios (1-5).

Las tierras raras presentes en los minerales que las contienen o en otros recursos secundarios se pueden lixiviar en distintos medios acuosos, por ejemplo: en nitrato, sulfato y cloruro. El primer elemento que pertenece a este grupo se descubrió en 1794. La tabla I muestra la lista cronológica del descubrimiento de las distintas tierras raras junto a su radio iónico estimado (6 y 7).

\section{PROCESOS DE SEPARACIÓN}

\subsection{Separación fotoquímica}

Mediante esta técnica, se ha estudiado la separación de europio de otras tierras raras (8). Se empleó como fuente de excitación una lámpara de mercurio de baja presión, con una longitud de onda de 185 $\mathrm{nm}$. Las tierras raras estaban presentes en una concentración de $0,01 \mathrm{M}$ (como percloratos binarios o ternarios) en un medio $0,05 \mathrm{M}$ de $\mathrm{K}_{2} \mathrm{SO}_{4}$ con un 10 $\%$ de isopropanol. La irradiación del Eu(III) en su banda de transferencia de carga se puede representar mediante:

$$
\mathrm{Eu}^{3+}\left(\mathrm{H}_{2} \mathrm{O}\right) \rightarrow \mathrm{Eu}^{2+}+\mathrm{H}^{+}+\bullet \mathrm{OH}
$$


TABla I.- Cronología del descubrimiento y radio iónico de las tierras raras

TABLE I.- Rare earths chronology and ionic radii

\begin{tabular}{|c|c|c|c|}
\hline Año & Elemento & Descubridor & $\begin{array}{c}\text { Radio iónico, } \\
\AA^{*}\end{array}$ \\
\hline 1794 & Itrio & Gadolin & 0,900 \\
1814 & Cerio & Berzelius & 1,010 \\
1839 & Lantano & Mosander & 1,045 \\
1843 & Erbio & Mosander & 0,890 \\
1878 & Terbio & Mosander & 0,923 \\
1878 & Yterbio & Marignac & 0,868 \\
1879 & Samario & de Boisbaudran & 0,958 \\
1879 & Escandio & Nilson & 0,745 \\
1879 & Holmio & Cleve & 0,901 \\
1879 & Tulio & Cleve & 0,88 \\
1880 & Gadolinio & Marignac & 0,938 \\
1885 & Praseodimio & von Welsbach & 0,997 \\
1885 & Neodimio & von Welsbach & 0,983 \\
1886 & Disprosio & de Boisbaudran & 0,912 \\
1896 & Europio & Demarçay & 0,947 \\
1907 & Lutecio & Urbain & 0,861 \\
1945 & Prometio & Glendenin/Marinsky & 0,97 \\
\hline
\end{tabular}

* Elemento como $\mathrm{Me}^{3+}$ y coordinación de 6 .

El isopropanol puede "retirar" el radical libre formado en la reacción anterior según:

$$
\left(\mathrm{CH}_{3}\right)_{2}+\bullet \mathrm{OH} \rightarrow \mathrm{H}_{2} \mathrm{O}+\left(\mathrm{CH}_{3}\right)_{2} \bullet \mathrm{COH}
$$

El Eu(II) formado se puede separar de la disolución precipitándolo como $\mathrm{EuSO}_{4}$. Mediante este método, se pueden conseguir factores de separación de $1(\mathrm{Eu} / \mathrm{Pr})$ y mayores de $200(\mathrm{Eu} / \mathrm{Tm})$.

También se ha considerado la separación del europio de una mezcla compuesta por $\mathrm{SmCl}_{3}$, $\mathrm{EuCl}_{3}$ y $\mathrm{GdCl}_{3}$ que estaba presente en un medio etanol-isopropanol; en este caso, la fotorreducción del europio se llevaba a cabo mediante una lámpara de mercurio de alta presión (9).

El proceso de separación consiste en una serie de reacciones:

a) Proceso de disolución y complejación

$$
\begin{aligned}
& (\mathrm{Sm}, \mathrm{Eu}, \mathrm{Gd}) \mathrm{Cl}_{3} \cdot n \mathrm{H}_{2} \mathrm{O}+3 \mathrm{EtOH} \rightarrow \\
& \rightarrow(\mathrm{Sm}, \mathrm{Eu}, \mathrm{Gd}) \mathrm{Cl}_{3} \cdot 3 \mathrm{EtOH}+n \mathrm{H}_{2} \mathrm{O}
\end{aligned}
$$

$(\mathrm{Sm}, \mathrm{Eu}, \mathrm{Gd}) \mathrm{Cl}_{3} \cdot \mathrm{nH}_{2} \mathrm{O}+3$ (isoprop) $\rightarrow$

$\rightarrow(\mathrm{Sm}, \mathrm{Eu}, \mathrm{Gd}) \mathrm{Cl}_{3} \cdot 3$ (isoprop) $+\mathrm{nH}_{2} \mathrm{O}$

b) Fotoexcitación

$$
\begin{aligned}
& (\mathrm{Sm}, \mathrm{Eu}, \mathrm{Gd}) \mathrm{Cl}_{3} \cdot 3 \mathrm{EtOH} \rightarrow \\
& \rightarrow(\mathrm{Sm}, \mathrm{Eu}, \mathrm{Gd}) \bullet \mathrm{Cl}_{3} \cdot 3 \mathrm{EtOH}
\end{aligned}
$$

$$
\begin{gathered}
(\mathrm{Sm}, \mathrm{Eu}, \mathrm{Gd}) \mathrm{Cl}_{3} \cdot 3 \text { (isoprop) } \rightarrow(\mathrm{Sm}, \mathrm{Eu}, \mathrm{Gd}) \\
\bullet \mathrm{Cl}_{3} \cdot 3 \text { (isoprop) }
\end{gathered}
$$

c) Transición electrónica

$$
\mathrm{Eu} \bullet \mathrm{Cl}_{3} \cdot 3 \mathrm{EtOH} \rightarrow \mathrm{EuCl}_{2}+2 \mathrm{EtOH}+\text { otros }
$$

$\mathrm{Eu} \bullet \mathrm{Cl}_{3} \cdot 3$ (isoprop) $\rightarrow \mathrm{EuCl}_{2}+2$ (isoprop) + otros

La separación del europio es posible ya que los potenciales de reducción del samario y del Gd(III) son más negativos que los del par Eu(III) o Eu(II). Ésto hace que sólo la reducción del europio sea posible aunque tanto el samario como el gadolinio se exciten de acuerdo a las ecs. [5] y [6].

\section{d) Precipitación}

El $\mathrm{EuCl}_{2}$ se precipita y separa de las otras tierras raras debido a la baja solubilidad de esta sal en la mezcla EtOH-isopropanol. Es muy importante mantener muy bajo el contenido de agua de esta mezcla con el fin de precipitar al $\mathrm{EuCl}_{2}$.

\subsection{Cambio de ion}

Esta técnica también se ha utilizado para la separación de las tierras raras. Se ha estudiado la separación y purificación de microcantidades de $\mathrm{Sc}$ (III) de macrocantidades de otras tierras raras (10). En este caso, se ensayaron distintos intercambiadores iónicos como geles fosfónicos, derivados aminofosfónicos y mezclas celulosa-fosfato.

Los procesos de cambio de ion aplicados a la separación-recuperación de estos elementos pueden constar de múltiples columnas para conseguir, como se ha mencionado, el objetivo final que es la obtención por separado de productos con un máximo de pureza. La tabla II muestra algunos valores típicos obtenidos de esta operación (11).

\subsection{Extracción supercrítica}

La síntesis de los carbonatos de lantanio, neodimio, samario, europio, gadolinio, disprosio y holmio (todos en estado de oxidación (III)), es posible mediante la reacción de una suspensión acuosa de los óxidos con $\mathrm{CO}_{2}$ a una temperatura igual o superior a $31{ }^{\circ} \mathrm{C}$ y a una presión de 72,9 atm. En estas condiciones, los carbonatos de $\operatorname{Pr}(\mathrm{III}), \mathrm{Tb}(\mathrm{III})$, $\mathrm{Er}(\mathrm{III})$ e $\mathrm{Yb}$ (III), o bien no se forman o lo hacen con un rendimiento muy bajo (12).

Esta técnica también se ha utilizado en la separación de los óxidos o hidróxidos lantánidos (en 
TABLA II.- Rendimiento y pureza típicos de los óxidos de tierras raras obtenidos mediante cambio de ion

TABLE II.- Tipical yields and purities of the rare earth oxides recovered through ion exchange

\begin{tabular}{|c|c|c|}
\hline Óxido metálico & Rendimiento, \% & Pureza, \% \\
\hline $\mathrm{Er}_{2} \mathrm{O}_{3}$ & 82 & 99,9 \\
$\mathrm{Yb}_{2} \mathrm{O}_{3}$ & 50 & 99,9 \\
$\mathrm{Tm}_{2} \mathrm{O}_{3}$ & 86 & 97 \\
$\mathrm{Ho}_{2} \mathrm{O}_{3}$ & 66 & 98 \\
$\mathrm{Dy}_{2} \mathrm{O}_{3}+\mathrm{Y}_{2} \mathrm{O}_{3}$ & s.d. & 99,9 \\
\hline
\end{tabular}

estado III) de los correspondientes lantánidos (en estado IV) (13). En este caso, las condiciones de operación son $40{ }^{\circ} \mathrm{C}$ y 100 atm., formándose después de $1 \mathrm{~h}$ de reacción los carbonatos de lantano, neodimio, samario, europio, gadolinio, holmio, prometio y tulio (en estado III) con un rendimiento superior al $95 \%$. Se obtiene como resultado un sólido que contiene los carbonatos y los materiales que no han reaccionado. La adición de $\mathrm{HCl} 0,5 \mathrm{M}$ al sólido a temperatura y presión ambientes, hace que se solubilicen los carbonatos formados y así se pueden separar las tierras raras disueltas mediante extracción con disolventes o cambio de ion.

\subsection{Extracción con disolventes}

Esta técnica se ha empleado con asiduidad para la separación y purificación de las tierras raras (1416).

El esquema de separación convencional que se sigue es la lixiviación del mineral o concentrado y el empleo de la disolución cargada que contiene las tierras raras como alimentación de la etapa de extracción con disolventes.

Con esta etapa se pretende separar en distintas corrientes los elementos considerados como ligeros (lantanio, cerio, proseodimio y neodimio), medios (samario, europio y gadolinio) y pesados (terbio, disprosio, holmio, erbio, tulio, iterbio, lutecio e itrio). Una vez conseguida esta separación, el paso siguiente consiste en emplear otras corrientes de extracción que permitan la separación de cada uno de estos elementos.

La última purificación, que permite la obtención de cada una de las tierras raras con una pureza muy elevada, se lleva a cabo mediante las técnicas de cambio de ion o cromatográficas.

\subsubsection{Agentes de extracción ácidos}

Como agentes de extracción ácidos se han empleado los ácidos carboxílicos que presentan la ventaja de ser relativamente baratos y asequibles, siendo su solubilidad en los medios acuosos el principal inconveniente.

La reacción general que representa la extracción de un metal por un ácido carboxílico (considerando la especie dímera del ácido) es:

$$
M e_{\mathrm{ac}}^{\mathrm{n}+}+\frac{1}{2} m 2(H R)_{2_{\text {org }}} \Leftrightarrow M e R_{\mathrm{n}}(H R)_{\mathrm{m}-\mathrm{n}_{\text {org }}}+n H_{\mathrm{ac}^{+}}
$$

Entre los ácidos carboxílicos empleados se encuentran el ácido versático 10, ácidos nafténicos y el ácido 2-bromodecanoico, todos diluidos en xileno (17). El orden de extracción encontrado es $\mathrm{La}<\mathrm{Ce}<\mathrm{Nd}<\mathrm{Gd}<\mathrm{Y}<\mathrm{Ho}<\mathrm{Yb}$ para el ácido versático 10 , y $\mathrm{La}<\mathrm{Ce}<\mathrm{Y}<\mathrm{Nd}<\mathrm{Gd}=\mathrm{Ho}=\mathrm{Yb}$ para los ácidos nafténicos; el ácido bromodecanoico extrae las tierras raras a valores de $\mathrm{pH}$ más ácidos (1-2) que los otros dos agentes de extracción.

La extracción de los nitratos de itrio y de los lantánidos trivalentes también se ha estudiado empleando ácidos carboxílicos diluidos en xileno (18), que presentaban distintos sustituyentes alquílicos en su molécula. El efecto estérico de la molécula del ácido influye en la extracción de los lantánidos y está relacionada con el número atómico del elemento metálico.

Para ácidos carboxílicos que presentan ramificaciones en las cadenas alquílicas, el equilibrio de extracción es:

$$
L n_{\mathrm{ac}}^{3+}+3(H R)_{2_{\text {org }}} \Leftrightarrow \operatorname{LnR}_{3_{\text {org }}}+3 H_{\mathrm{ac}}^{+}
$$

$\mathrm{El}$ valor del $\mathrm{pH}_{50}$ disminuye en la serie $\mathrm{La}-\mathrm{Lu}$, es decir, en el mismo orden en el que disminuye el radio iónico con el aumento del número atómico. El itrio se comporta de forma parecida al gadolinio y al terbio, que son tierras raras consideradas como medias.

Cuando el ácido carboxílico no presenta ramificaciones en sus cadenas alquílicas, el valor del $\mathrm{pH}_{50}$ varía desde el lantano hasta un mínimo para las tierras raras ligeras y luego aumenta hasta el lutecio. En este caso, el itrio se comporta de forma parecida a las tierras raras ligeras.

El equilibrio de extracción para las tierras raras ligeras y medias se puede representar según:

$$
2 L n_{\mathrm{ac}}^{3+}+6(H R)_{2_{\text {org }}} \Leftrightarrow\left(\operatorname{LnR}_{3}(H R)_{3}\right)_{2_{\text {org }}}+6 H_{\mathrm{ac}}^{+}
$$

Las tierras raras pesadas se extraen según:

$$
2 L n_{\mathrm{ac}}^{3+}+5(H R)_{2_{\text {org }}} \Leftrightarrow\left(\operatorname{Ln} R_{3}(H R)_{3}\right)_{2_{\text {org }}}+4 H_{\mathrm{ac}}^{+}
$$


Otra clase de agentes de extracción de tipo ácido que se ha utilizado para la separación de las tierras raras es la de los ácidos alquilfosfóricos y, entre ellos, el más empleado ha sido el DEHPA (ácido di2-etilhexilfosfórico).

Este ácido se ha usado diluido en queroseno para estudiar los factores de separación de las distintas tierras raras (19). Se observa que la extracción de estos elementos mejora en el orden $\mathrm{La}<\mathrm{Ce}$ $<\mathrm{Nd}<\mathrm{Sm}<\mathrm{Eu}<\mathrm{Gd}<\mathrm{Tb}<\mathrm{Dy}=\mathrm{Y}<\mathrm{Ho}<\mathrm{Er}<$ $\mathrm{Tm}<\mathrm{Yb}<\mathrm{Lu}$, disminuyendo el valor del coeficiente de distribución con el aumento de la temperatura. En la figura 1 se representan los valores de los factores de separación entre elementos contiguos pertenecientes al grupo de las tierras raras, mientras que en la tabla III se muestra otra serie de valores de factores de separación entre estos elementos. En todos los casos, las extracciones se llevaron a cabo en un medio $\mathrm{HCl} 0,1 \mathrm{M}$ mediante una disolución de DEHPA 0,2M en queroseno.

Otro ácido empleado ha sido el EHEHPA (ácido 2-etilhexil-2 etilhexilfosfórico), también diluido en

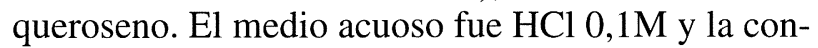
centración del agente de extracción en queroseno $0,2 \mathrm{M}$. En este sistema, la extracción del metal aumenta con el número atómico aunque es menor si se compara con el sistema del DEHPA.

Aunque el DEHPA se puede utilizar en la separación de las tierras raras, dicha separación entre elementos contiguos se ve favorecida cuando se emplean ácidos fosfónicos o fosfínicos (20).

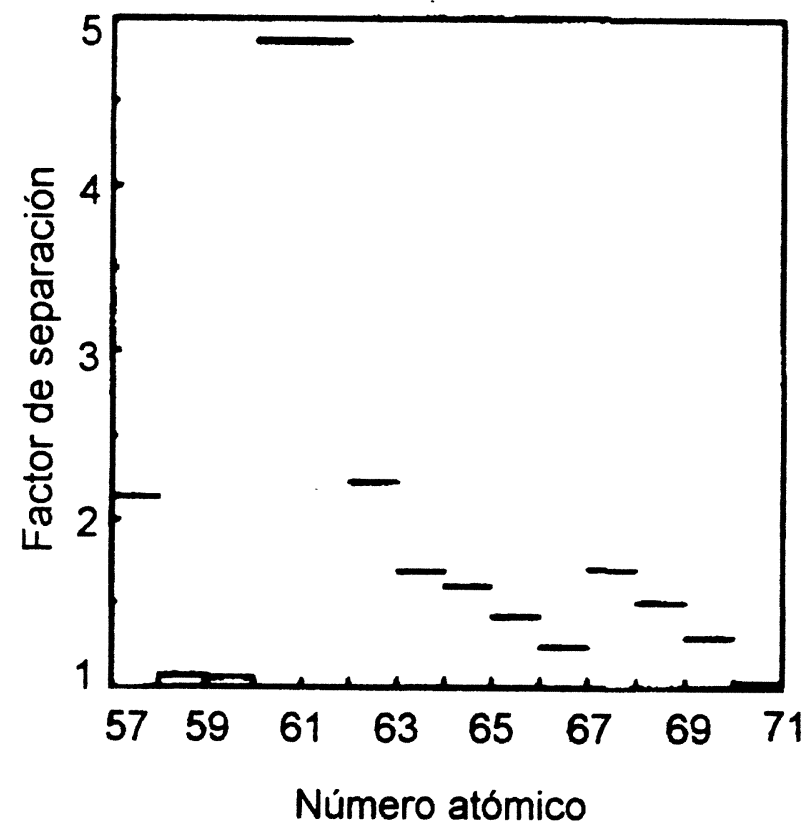

FIG. 1.- Factores de separación ( $\gamma_{\text {núm. atómico }}$ inf./núm. atómico sup.) entre elementos pertenecientes al grupo de las tierras raras.

FIG. 1.- Separation factors ( $\gamma_{\text {low atomic number/high }}$ atomic number) of rare earth elements.
TABLA III.- Valores de los factores de separación para elementos pertenecientes al grupo de las tierras raras

TABLE III.- Separation factors of rare earth elements

\begin{tabular}{|c|c|c|c|c|c|}
\hline $\begin{array}{c}\text { Elemento } \\
A / B\end{array}$ & $\gamma_{\mathrm{A} / \mathrm{B}}$ & $\begin{array}{c}\text { Elemento } \\
A / B\end{array}$ & $\gamma_{\mathrm{A} / \mathrm{B}}$ & $\begin{array}{c}\text { Elemento } \\
A / B\end{array}$ & $\gamma_{\mathrm{A} / \mathrm{B}}$ \\
\hline La-Gd & 44,6 & Nd-Gd & 18,3 & Gd-Tm & 7,15 \\
La-Ho & 125 & Nd-Ho & 51,3 & Gd-Yb & 9,30 \\
La-Tm & 319 & Nd-Tm & 131 & Gd-Lu & 9,55 \\
La-Yb & 414 & Nd-Yb & 170 & Tb-Ho & 1,76 \\
La-Lu & 425 & Nd-Lu & 175 & Tb-Tm & 4,48 \\
Ce-Gd & 20,9 & Sm-Gd & 3,75 & Tb-Yb & 5,83 \\
Ce-Ho & 58,4 & Sm-Ho & 10,6 & Tb-Lu & 5,90 \\
Ce-Tm & 149 & Sm-Tm & 27,0 & Dy-Tm & 3,16 \\
Ce-Yb & 193 & Sm-Yb & 35,1 & Dy-Yb & 4,11 \\
Ce-Lu & 199 & Sm-Lu & 36,0 & Dy-Lu & 4,22 \\
Pr-Gd & 19,5 & Eu-Ho & 4,74 & Ho-Tm & 2,55 \\
Pr-Ho & 54,7 & Eu-Tm & 12,0 & Ho-Yb & 3,31 \\
Pr-Tm & 139 & Eu-Yb & 15,7 & Ho-Lu & 3,41 \\
Pr-Yb & 181 & Eu-Lu & 16,2 & Er-Yb & 1,90 \\
Pr-Lu & 186 & Gd-Ho & 2,80 & Er-Lu & 2,01 \\
\hline
\end{tabular}

El tercer tipo de reactivos ácidos que se ha empleado en la separación de estos elementos ha sido el de las hidroxioximas y, en general, los agentes de extracción ácidos capaces de formar compuestos de tipo quelato. Estos agentes de extracción se emplean tradicionalmente para la del $\mathrm{Cu}(\mathrm{II}) \mathrm{de}$ distintos medios acuosos (21-23), aunque cada vez son más los estudios en los que se amplia su campo de utilización a otros metales.

Por ejemplo, se ha empleado el SME 529 (actualmente LIX 84) para estudiar el equilibrio de extracción del $\mathrm{Ce}$ (III) y $\mathrm{La}$ (III) en un medio cloruro sódico, diluyéndose el agente de extracción en n-heptano (24).

El equilibrio de extracción depende del metal; así, para el caso del cerio:

$$
C e_{a c}^{3+} 3 H R_{\text {org }} \Leftrightarrow C e R_{3_{\text {org }}}+3 H_{\mathrm{ac}}^{+}
$$

mientras que en el caso del lantano la extracción se representa mediante:

$\mathrm{La}_{\mathrm{ac}}^{3+}+2 \mathrm{HR}_{\mathrm{org}}+\mathrm{H}_{2} \mathrm{O} \Leftrightarrow \mathrm{LaR}_{2} \mathrm{OH}_{\mathrm{org}}+3 \mathrm{H}_{\mathrm{ac}}^{+}$

También se ha empleado el LIX 70 para la extracción de estas dos tierras raras (25). En este caso, como diluyentes de la fase orgánica se ensayaron el queroseno y el $\mathrm{n}$-heptano, siendo el medio acuoso $\mathrm{NaCl}$.

Tanto el cerio como el lantano se extraen cuantitativamente, aunque el cerio se puede extraer a valores de $\mathrm{pH}$ más bajos. Con este reactivo, el equilibrio de extracción para ambos metales se puede representar mediante: 


$$
M e_{\mathrm{ac}}^{3+}+3 H R_{\mathrm{org}} \Leftrightarrow M e R_{3 \mathrm{org}}+3 H_{\mathrm{ac}}^{+}
$$

En ambos sistemas (SME 529 y LIX 70), el equilibrio de extracción correspondiente se alcanza, aproximadamente, a los 2 min de contacto entre ambas fases.

Otro tipo de agentes de extracción capaz de formar compuestos de tipo quelato es el de los derivados de la quinolina (oxinas) y, entre ellos, el Kelex 100 diluido en n-heptano y $10 \% \mathrm{v} / \mathrm{v}$ de $\mathrm{n}$-decanol se ha utilizado para la extracción de Ce(III) y $\mathrm{La}$ (III) de disoluciones de $\mathrm{NaCl}$ (26). Para cada uno de estos metales, el equilibrio de extracción responde a una reacción como la mostrada en la ec. [15]. Sin embargo, también existe la posibilidad de que las especies hidrolizadas de lantano sean extraídas por este derivado de la oxina según:

$$
\mathrm{LaOH}_{\mathrm{ac}}^{2+}+3 H R_{\mathrm{org}} \Leftrightarrow \mathrm{LaR}_{3_{\mathrm{org}}}+2 \mathrm{H}_{\mathrm{ac}}^{+}+\mathrm{H}_{2} \mathrm{O}
$$

El LIX 54 (B-dicetona) diluido en n-heptano, también es un agente de extracción efectivo del $\mathrm{Ce}(\mathrm{III})$ y $\mathrm{La}(\mathrm{III})$ de una disolución acuosa de $\mathrm{NaCl}$ (27). En el caso del cerio, la extracción responde al equilibrio de la ec. [15], mientras que para el lantano, la reacción es:

$$
L a_{\mathrm{ac}}^{3+}+6 H R_{\mathrm{org}} \Leftrightarrow L a R_{3} \cdot 3 H R_{\mathrm{org}}+3 H_{a c}^{+}
$$

\subsubsection{Aminas y sales de amonio cuaternario}

Se ha empleado una sal de amonio cuaternario $\left(\mathrm{R}_{3} \mathrm{CH}_{3} \mathrm{~N}^{+} \mathrm{NO}_{3}^{-}\right)$diluida en xileno para separar el praseodimio y el neodimio (28). La concentración de la sal de amonio fue $0,65 \mathrm{M}$. La reacción de extracción se representa mediante el equilibrio:

$$
\begin{aligned}
\mathrm{Me}_{\mathrm{ac}}^{3+} & +3 \mathrm{NO}_{3_{\mathrm{ac}}^{-}}+\frac{3}{2}\left(\mathrm{R}_{3} \mathrm{CH}_{3} \mathrm{~N}^{+} \mathrm{NO}_{3}^{-}\right)_{2_{\text {org }}} \Leftrightarrow \\
& \Leftrightarrow \mathrm{MeNO}_{3} \cdot 3 \mathrm{R}_{3} \mathrm{CH}_{3} \mathrm{~N}^{+} \mathrm{NO}_{3_{\text {org }}^{-}}
\end{aligned}
$$

El electrólito presente en el medio acuoso influye sobre la extracción de las tierras raras, especialmente sobre las consideradas ligeras, habiéndose estudiado dicha influencia con el empleo de Aliquat 336 (sal de amonio) diluida en diluyentes aromáticos o alifáticos y variando la concentración de $\mathrm{NH}_{4} \mathrm{NO}_{3}$ en el medio acuoso de 4 a $8 \mathrm{M}$ (29).

En este sistema, la extracción y separación de las tierras raras ligeras se atribuye a un mecanismo de extracción complejo en el que intervienen tres equilibrios de extracción.

Cuando la concentración del metal es baja, el equilibrio de extracción se representa mediante:

$$
\begin{aligned}
& n R_{3} \mathrm{CH}_{3} \mathrm{~N}^{+} \mathrm{NO}_{3_{\text {org }}^{+}}^{+}+\mathrm{Me}\left(\mathrm{NO}_{3}\right)_{3_{\text {ac }}} \Leftrightarrow \\
& \Leftrightarrow\left(\mathrm{R}_{3} \mathrm{CH}_{3} \mathrm{~N}^{+} \mathrm{NO}_{3}^{-}\right)_{\mathrm{n}} \cdot \mathrm{Me}\left(\mathrm{NO}_{3}\right)_{3_{\text {org }}}
\end{aligned}
$$

disminuyendo el coeficiente $n$ al aumentar la carga del metal en la fase orgánica.

Existe la posibilidad de que se formen especies metálicas aniónicas:

$$
\mathrm{Me}^{3+}+4 \mathrm{NO}_{3}^{-} \Leftrightarrow \mathrm{Me}\left(\mathrm{NO}_{3}\right)_{4}^{-}
$$

El catión de la sal de amonio disociada reacciona en la fase acuosa con la especie $\mathrm{Me}\left(\mathrm{NO}_{3}\right)_{4}^{-}$; de esta forma, el complejo formado se transfiere a la fase orgánica según:

$$
\begin{gathered}
\mathrm{R}_{3} \mathrm{CH}_{3} \mathrm{~N}^{+} \mathrm{NO}_{3_{\text {org }}^{-}}^{-}+\mathrm{Me}\left(\mathrm{NO}_{3}\right)_{4}^{-} \Leftrightarrow \mathrm{R}_{3} \mathrm{CH}_{3} \mathrm{~N}^{+} . \\
\mathrm{Me}\left(\mathrm{NO}_{3}\right)_{4_{\text {org }}}^{-}+\mathrm{NO}_{3_{\mathrm{ac}}}^{-}
\end{gathered}
$$

Este complejo 1:1 reacciona posteriormente con otra molécula de $\mathrm{Me}\left(\mathrm{NO}_{3}\right)_{3}$, con lo que se forma un complejo metal:sal de amonio 2:1 en la fase orgánica:

$$
\begin{gathered}
\mathrm{R}_{3} \mathrm{CH}_{3} \mathrm{~N}^{+} \mathrm{Me}\left(\mathrm{NO}_{3}\right)_{4_{\mathrm{org}}}^{-}+\mathrm{Me}\left(\mathrm{NO}_{3}\right)_{3_{\mathrm{ac}}} \Leftrightarrow \\
\Leftrightarrow \mathrm{R}_{3} \mathrm{CH}_{3} \mathrm{~N}^{+} \mathrm{Me}\left(\mathrm{NO}_{3}\right)_{4}^{-} \mathrm{Me}\left(\mathrm{NO}_{3}\right)_{3_{\text {org }}}
\end{gathered}
$$

A su vez, el $\mathrm{Me}\left(\mathrm{NO}_{3}\right)_{3}$, en las reacciones anteriores, está en equilibrio con los iones $\mathrm{Me}^{3+}$ y $\mathrm{NO}_{3}^{-}$:

$$
\mathrm{Me}^{3+}+\mathrm{NO}_{3}^{-} \Leftrightarrow \mathrm{Me}\left(\mathrm{NO}_{3}\right)_{3}
$$

aumentando el electrólito de soporte $\left(\mathrm{NH}_{4} \mathrm{NO}_{3}\right)$ la concentración de iones $\mathrm{NO}_{3}^{-}$en el medio:

$$
\mathrm{NH}_{4}^{+}+\mathrm{NO}_{3}^{-} \Leftrightarrow \mathrm{NH}_{4} \mathrm{NO}_{3}
$$

con lo que todos los equilibrios anteriores (ecs. [19] a [23]) se desplazan a la derecha.

La tabla IV muestra los valores de los factores de separación entre distintas tierras raras cuando se emplea como fase orgánica Aliquat 336 diluido en Shellsol AB (30). Se observa que para el par Pr-Nd el factor de separación obtenido es mayor que los conseguidos para cualquier otro par de elementos adyacentes.

El tipo de amina y el medio acuoso influyen decisivamente en la extracción de estos elementos. Las aminas primarias $\left(\mathrm{RNH}_{2}\right)$ extraen estos metales de un medio sulfato, mientras que las aminas terciarias $\left(\mathrm{R}_{3} \mathrm{~N}\right)$ los extraen de medio nitrato. En general, las tierras raras ligeras se extraen preferentemente con estos agentes de extracción básicos. 
TABLA IV.- Valores de los factores de separación entre tierras raras contiguas

TABLE IV.- Values of separation factors between adjacents rare earths

\begin{tabular}{|c|c|}
\hline Metal $A-B$ & Factor de separación $\left(\gamma_{\mathrm{A} / \mathrm{B}}\right)$ \\
\hline La-Ce & 1,67 \\
Ce-Pr & 1,72 \\
Pr-Nd & 2,04 \\
Sm-Eu & 1,42 \\
Eu-Gd & 1,75 \\
Gd-Tb & 1,02 \\
Tb-Dy & 1,08 \\
Dy-Ho & 1,24 \\
Ho-Er & 1,43 \\
Er-Tm & 1,59 \\
Tm-Yb & 1,47 \\
Yb-Lu & 1,34 \\
\hline
\end{tabular}

F. Orgánica: 0,5M Aliquat 336 en ciclo nitrato.

F. Acuosa: 0,02M elemento metálico en nitrato sódico 4M. Temperatura: $20^{\circ} \mathrm{C}$.

\subsubsection{Agentes de extracción neutros}

Como en el caso de otros metales, el TBP (tributilfosfato) se ha empleado para extraer las tierras raras de distintos medios acuosos.

Este agente de extracción y el DBBP (di-n-butil n-butilfosfonato) extraen los lantánidos mediante la siguiente reacción:

$$
L n_{\mathrm{ac}}^{3+}+3 N O_{3_{\mathrm{ac}}}^{-}+3 L_{\mathrm{org}} \Leftrightarrow \operatorname{Ln}\left(N \mathrm{O}_{3}\right)_{3} L_{3_{\mathrm{org}}}
$$

donde $L$ representa al agente de extracción (31). Con estos dos agentes de extracción, se obtienen preferentemente los elementos intermedios de esta serie, especialmente el samario y el europio.

Otro agente de extracción utilizado ha sido el P350 (di(1-metil-heptil)metilfosfonato) diluido en queroseno o en hexano. El metal estudiado fue el neodimio y se emplearon distintos medios acuosos (32). El mecanismo de extracción se puede representar por la ecuación:

$$
\begin{gathered}
\mathrm{Nd}_{\mathrm{ac}}^{3+}+3 X_{\mathrm{ac}}^{-}+n(R O)_{2} \mathrm{CH}_{3} P O_{\mathrm{org}} \Leftrightarrow \\
\Leftrightarrow N d X_{3} \cdot n(R O)_{2} \mathrm{CH}_{3} P O_{\mathrm{org}}
\end{gathered}
$$

donde $X^{-}$representa a $\mathrm{SCN}^{-}, \mathrm{ClO}_{4}^{-}$o a $\mathrm{NO}_{3}^{-}$. $\mathrm{La}$ extracción responde a un mecanismo por solvatación de la especie $\mathrm{NdX}_{3}$, compitiendo favorablemente el agente de extracción para sustituir a las moléculas de agua coordinadas a la especie metálica.

\subsubsection{Agentes de extracción macrocíclicos}

Se han empleado éteres corona (sim-dibenzo-16corona-5-ácido oxiacético) como agentes de extracción de los elementos lantánidos (33). Este éter se disolvió en una mezcla 80:20 cloroformo-heptanol. La extracción de las diversas tierras raras depende del $\mathrm{pH}$ del medio acuoso y, así, el $\mathrm{Lu}^{3+}$ se extrae cuantitativamente a un $\mathrm{pH}$ de 6,7 , mientras que el $\mathrm{La}^{3+}, \mathrm{Pr}^{3+}, \mathrm{Sm}^{3+}, \mathrm{Eu}^{3+}, \mathrm{Tb}^{3+}, \mathrm{Er}^{3+}$ e $\mathrm{Yb}^{3+}$ lo hacen casi cuantitativamente $(>98 \%)$ a un $\mathrm{pH}$ de 6,5 . La estequiometría del complejo extraído es $1: 2$ (metal:agente de extracción).

Otro sistema de separación de estos elementos ha sido el que emplea un ligando macrocíclico junto a un agente de extracción como la HTTA (tenoiltrifluoracetona) (34). Como ligandos se ensayaron DAPDA (1,7-diaza-4,10,13-trioxa-ciclopentadecano-N,N'-ácido diacético) y DACDA $(1,10-$ diaza-4,7,13,16-tetraoxa-ciclooctadecano-N,N'-ácido diacético). La extracción de los lantánidos responde al equilibrio:

$$
L n_{\mathrm{ac}}^{3+}+3 H R_{\text {org }} \Leftrightarrow L n R_{3_{\text {org }}}+3 H_{\text {ac }}^{+}
$$

donde $H R$ representa la forma protonada del HTTA. El diluyente del medio orgánico influye en la extracción de los metales y, tanto en el caso del DAPDA como en el del DACDA, se ve favorecida en el orden nitrobenceno $>$ benceno $>$ cloroformo. Esta influencia se explica debido a que en diluyentes elevadamente polares se forman pares iónicos con la estequiometría $\mathrm{Lu}(\mathrm{DAPDA})^{+} \mathrm{TTA}^{-}$, que no se forman en diluyentes no polares.

El reactivo HTTA se ha utilizado junto al K22DD (4,13-didecil-1,7,10,16-tetraoxa-4,13-diazaciclooctadecano) disueltos en cloroformo para la extracción de $\mathrm{Am}^{3+}, \mathrm{Cm}^{3+}, \mathrm{Eu}^{3+}, \mathrm{Ce}^{3+}$ y $\mathrm{Pm}^{3+}$ de un medio acetato a un $\mathrm{pH}$ de 4,8 (35).

El mecanismo de extracción se representa mediante el equilibrio:

$$
\begin{gathered}
M e_{\mathrm{ac}}^{3+}+3 H T T A_{\mathrm{org}}+K 22 D D_{\mathrm{org}} \Leftrightarrow \\
\Leftrightarrow M e(T T A)_{3} K 22 D D_{\mathrm{org}}+3 H_{\mathrm{ac}}^{+}
\end{gathered}
$$

El empleo de esta mezcla aumenta el valor del coeficiente de distribución del metal en un factor entre $10^{4}$ y $10^{5}$.

Los calixarenos son $(1, \mathrm{n})$-ciclofanos que presen$\tan$ una cavidad formada por grupos fenilo que actúan como puentes, permitiendo, además, la introducción de sustituyentes en el esqueleto orgánico. Esta estructura ha hecho que estos compuestos se hayan estudiado como reactivos para la extracción de algunos lantánidos trivalentes (lantano, neodimio, europio, erbio e iterbio) (36). 
Se han empleado tres de estos reactivos cuya composición se muestra en la tabla V. Los tres calixarenos presentan distintos tamaños en sus cavidades, además de tener en su molécula grupos ácido carboxílico con el fin de favorecer un número de coordinación mayor para el metal a extraer.

En el caso de $\mathrm{C} 1$ disuelto en cloroformo, el orden de extracción es $\mathrm{Nd}, \mathrm{Eu}>\mathrm{La}>\mathrm{Er}>\mathrm{Yb}$ para un valor de $\mathrm{pH}$ de 2-3,5. El mecanismo de extracción es por intercambio catiónico, formándose un complejo 1:2 (metal:ligando) en la fase orgánica.

El ligando $\mathrm{C} 2$ presenta una cavidad más pequeña en su molécula, por lo que los coeficientes de distribución que se obtienen son menores que con C1. El complejo formado presenta la misma estequiometría que la descrita para $\mathrm{C} 1$, siendo el orden de extracción $\mathrm{Eu}>\mathrm{Nd}>\mathrm{Yb}>\mathrm{Er}>\mathrm{La}$.

Por último, C3 es un ligando que extrae mejor las tierras raras pesadas, siendo el orden de extracción $\mathrm{Yb}>\mathrm{Er}, \mathrm{Eu}>\mathrm{Nd}>\mathrm{La}$, y el valor del $\mathrm{pH}$ de extracción igual a 3.

\subsubsection{Mezclas sinérgicas}

Se han experimentado diversas mezclas sinérgicas para extraer las tierras raras de distintos medios acuosos. El concepto de sinergismo se puede resumir como el empleo de una mezcla de dos o más agentes de extracción de forma que, en las mismas condiciones experimentales, la extracción del metal se ve favorecida con respecto a la que se obtiene con cada uno de los agentes de extracción considerados separadamente.

Una mezcla estudiada ha sido la compuesta por TBP y Aliquat 336 (37). Este sistema es uno de los mejores para la separación de samario y de gadolinio, presentando factores de separación mayores de 3. El electrólito soporte, $\mathrm{NH}_{4} \mathrm{NO}_{3}$, es extraído tanto por el TBP como por el Aliquat 336, pero no por la mezcla de ambos.

TABLA V.- Composición de algunos calixarenos empleados en la extracción de tierras raras

TABLE V.-Composition of some calixarenes used in the extraction of rare earths

\begin{tabular}{|l|c|}
\hline \multicolumn{1}{|c|}{ Composición } & Nombre de referencia* \\
\hline $\begin{array}{l}\text { p-tert-butilcalix/6/ } \\
\text { areno ácido } \\
\text { hexacarboxílico } \\
\begin{array}{l}\text { p-tert-butil/4/areno } \\
\text { ácido tetracarboxílico } \\
{[3,1,3,1] \text {-ciclofano }}\end{array}\end{array}$ & $\mathrm{C} 1$ \\
\hline
\end{tabular}

* En este trabajo
El DBTPA (ácido dibutilmonotiofosfórico) y la PHEN (1,10-fenantrolina) forman una mezcla más efectiva que la compuesta por el DDTPA (ácido didodecilmonotiofosfórico) y la PHEN para la extracción del Eu(III) en un medio acetato (38). La extracción responde al equilibrio:

$$
\begin{gathered}
E u_{\mathrm{ac}}^{3+} 3 H R_{\mathrm{org}}+P H E N_{\mathrm{org}} \Leftrightarrow E u R_{3} \\
\cdot P H E N_{\mathrm{org}}+3 H_{\mathrm{ac}}^{+}
\end{gathered}
$$

donde $H R$ representa a los ácidos DBTPA o DDTPA.

La mezcla formada por el TBP y el Aliquat 336 (en ciclo nitrato) se ha utilizado también para un estudio sistemático sobre la extracción de una serie de lantánidos e Y(III) (39). El diluyente de la fase orgánica tiene gran influencia en el efecto sinérgico de la mezcla, puesto que éste mejora con dodecano/xileno con respecto a heptano/xileno, hexano/ xileno o a xileno solo.

La complejidad de estos sistemas de extracción se demuestra por el hecho de la existencia de 2-3 especies en la fase orgánica; las estequiometrías se muestran en la tabla VI. La constante de estabilidad del complejo formado disminuye con el aumento del número atómico del metal.

Se ha estudiado un ácido fosfónico como el PC88A (ácido 2-etilhexilfosfónico) disuelto en n-heptano junto al DTPA (dietilamina-ácido pentaacético) para la extracción de lantánidos. Los factores de separación entre $\mathrm{Y} / \mathrm{Ho} / \mathrm{Er}$ son mayores al adicionar DTPA, mientras que la estequiometría de la especie extraída se representa por $\mathrm{LnR}_{3} \cdot 3 \mathrm{HR}(40)$.

Una nueva mezcla de reactivos empleada para la extracción de las tierras raras es la formada por reactivos tipo quelato $(H R)$ y agentes de extracción neutros $(L)$ (41). La extracción responde a distintos equilibrios. La siguiente ecuación muestra uno característico:

$$
\begin{aligned}
& M e_{\mathrm{ac}}^{3+}+(3+a) H R_{\mathrm{org}}+b L_{\mathrm{org}} \Leftrightarrow \\
& \Leftrightarrow M e R_{3} \cdot a H R \cdot b L_{\mathrm{org}}+3 H_{\mathrm{ac}}^{+}
\end{aligned}
$$

TABLA VI.- Estequiometrías propuestas para la extracción de lantánidos e Y(III) por la mezcla TBP-Aliquat 336

TABLE VI.- Proposed stoichiometries for lanthanides and $Y(I I I)$ extraction by the mixture TBP-Aliquat 336

\begin{tabular}{|c|c|c|}
\hline $\operatorname{TBP}\left(\mathrm{L}_{\mathrm{I}}\right)$ & Aliquat 336 $\left(\mathrm{L}_{\mathrm{II}}\right)$ & Especie extraída \\
\hline 2 & 1 & $\mathrm{~L}_{\mathrm{II}}^{+}\left(\mathrm{Ln}\left(\mathrm{NO}_{3}\right)_{4} \cdot 2 \mathrm{~L}_{\mathrm{I}}\right)^{-}$ \\
3 & 1 & $\mathrm{~L}_{\mathrm{II}}^{+}\left(\mathrm{Ln}\left(\mathrm{NO}_{3}\right)_{4} \cdot 3 \mathrm{~L}_{\mathrm{I}}\right)^{+}$ \\
1 & 2 & $\left(\mathrm{~L}_{\mathrm{II}}{ }^{+}\right)_{2}\left(\mathrm{Ln}\left(\mathrm{NO}_{3}\right)_{5} \cdot \mathrm{L}_{\mathrm{I}}\right)^{2-}$ \\
\hline
\end{tabular}


En el caso de un reactivo de tipo ácido derivado del ácido fosfórico $(H R)$ y de un agente de extracción neutro $(L)$, la extracción de los lantánidos responde al equilibrio:

$$
L n_{\mathrm{ac}}^{3+}+2(H R)_{2_{\text {org }}}+L_{\text {org }} \Leftrightarrow \operatorname{Ln}\left(H R_{2}\right) R_{2} \cdot L_{\text {org }}+3 H_{\mathrm{ac}}^{+}
$$

para las condiciones de dimerización del agente de extracción ácido.

\subsection{Precipitación (en la etapa de reextracción)}

En algunos casos, las condiciones de operación en la etapa de extracción pueden hacer que la recuperación de los elementos metálicos de las disoluciones de reextracción sea problemática; este hecho se puede deber también, en algunos casos, a la necesidad de un elevado consumo de agentes neutralizantes con los consiguientes problemas de diversa naturaleza que este hecho conlleva.

Una forma de evitar este problema puede ser la adición de una sal a la disolución de reextracción con el fin de que precipiten los sulfatos dobles de estos elementos, sin que sea necesaria una neutralización de la disolución.

Algunos ejemplos de utilización de esta técnica de separación se tienen en la precipitación de lantano y neodimio de una fase orgánica de DEHPA en queroseno (42). La disolución sulfúrica de reextracción contenía un sulfato (sódico, potásico o amónico) como agente precipitante. La precipitación sólo ocurre en la fase acuosa mediante un compuesto del tipo $\mathrm{xMe}_{2} \mathrm{SO}_{4} \mathrm{yLn}_{2}\left(\mathrm{SO}_{4}\right)_{3} \mathrm{nH}_{2} \mathrm{O}$.

Las mejores características con respecto a esta precipitación que presentan los elementos ligeros respecto a los pesados, hacen que sea posible la separación de unos y otros mediante este tipo de procedimiento (43).

\subsection{Procesos industriales}

La importancia de estos elementos en la industria moderna ha llevado a la implementación de una serie de procesos para la obtención por separado de cada uno de estos elementos con un elevado grado de pureza. El proceso Rhône-Poulec es un ejemplo clásico de esta secuencia de separación.

En general, casi todos estos procesos emplean la extracción con disolventes como técnica para concentrar y separar las distintas tierras raras. Pese a las buenas posibilidades que esta técnica presenta, la separación de los distintos elementos no es fácil $y$, en muchos casos, requiere una forma de operar cuyo esquema muy raramente se emplea en la obtención de otros metales que no sean las tierras raras.

La operación, llamada de reflujo con alimentación forzada, se muestra en la figura 2. Las condiciones de operación se resumen en la alimentación de la fase acuosa que contiene las tierras raras que se quieren separar en un punto cercano a la mitad del sistema de mezcladores-sedimentadores, y a la recirculación de las corrientes de salida. Con este sistema, se fuerza al aumento de concentración de los distintos componentes, prosiguiendo la operación hasta que se consigue el grado de separación deseado. En ese momento, se para el proceso y se vacían los mezcladores-sedimentadores para obtener el producto deseado (44).

La figura 3 muestra un esquema de separación típico de lantano, neodimio y praseodimio utilizando TBP.

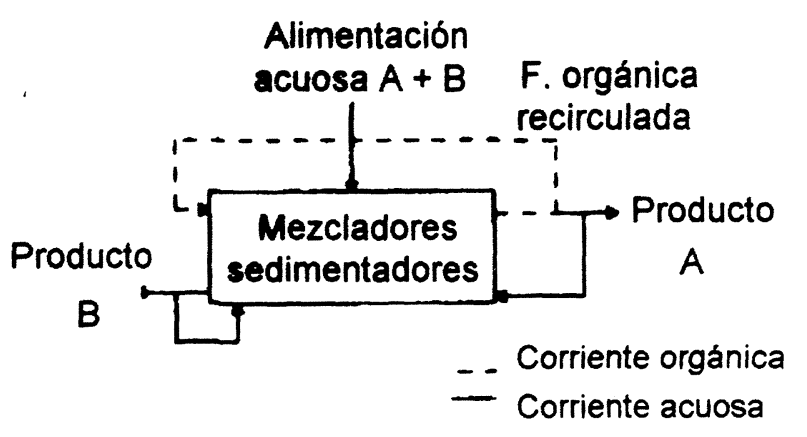

FIG. 2.- Sistema de mezcladores-sedimentadores bajo condiciones de reflujo con alimentación forzada.

FIG. 2.- Mixer-Settler bank under reflux configuration and forced-fed.

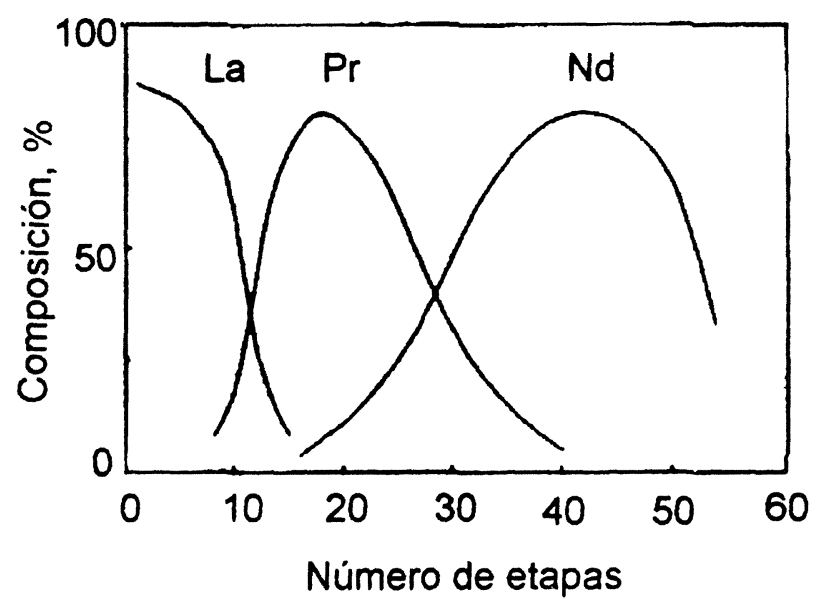

FIG. 3.- Perfiles de concentración en el sistema de mezcladores-sedimentadores bajo condiciones de reflujo.

FIG. 3.- Concentration profiles for mixer-settler operation under reflux. 


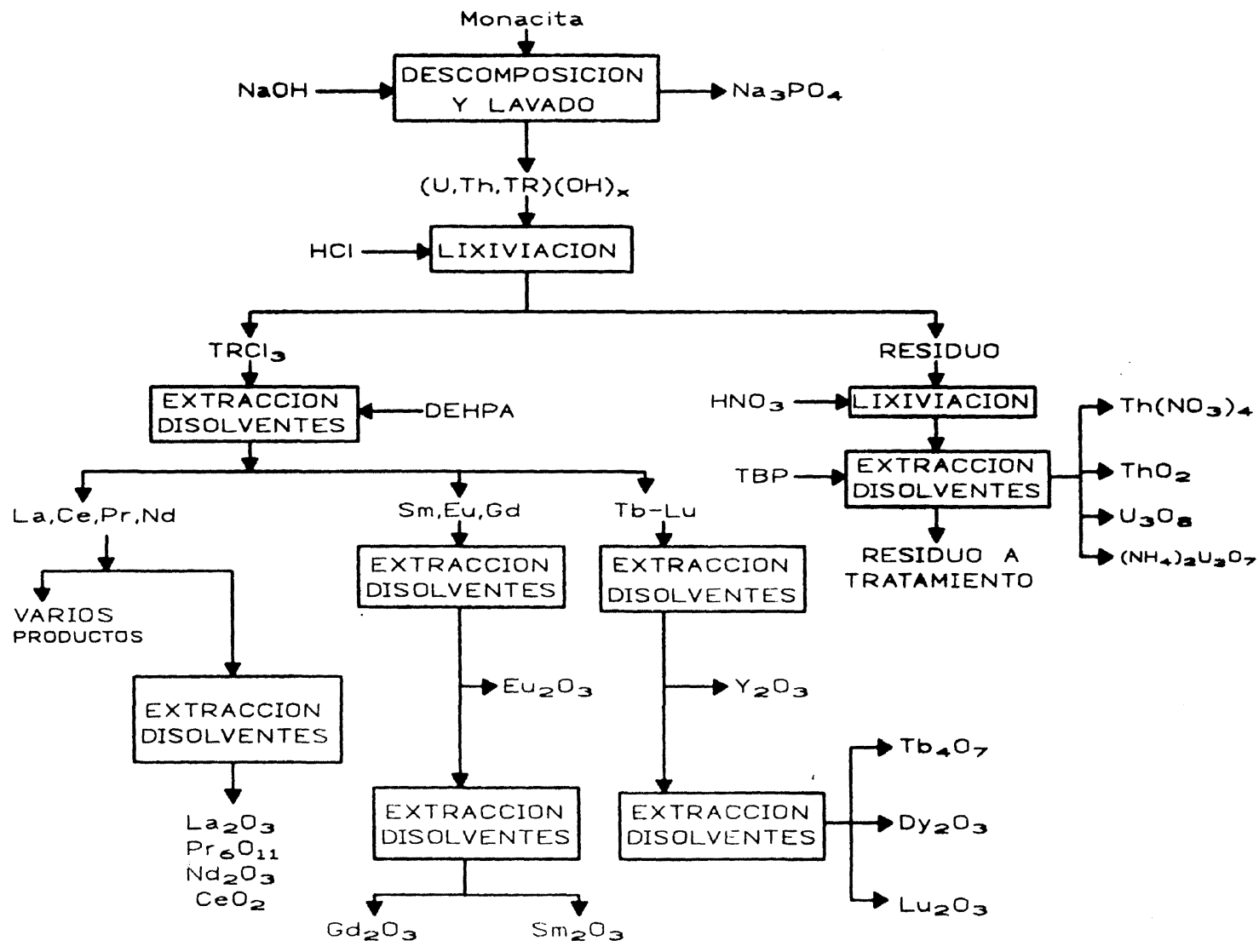

Fig. 4.- Diagrama de flujo simplificado de la planta Yao Lung (China).

FIG. 4.- Simplified flow-sheet of the Yao Lung plant (China).

En la actualidad, China posee la mayor reserva mundial de estos elementos, que se hallan en forma de óxidos (45). Una de las primeras plantas construidas para el beneficio de las tierras raras es la de Yao Lung. La figura 4 muestra el diagrama de flujo simplificado de la misma.

\section{Agradecimiento}

Los autores agradecen al CSIC la ayuda prestada para la realización del presente trabajo.

\section{Nomenclatura}

$\gamma_{\mathrm{A} / \mathrm{B}}=$ Factor de separación. Relación entre los coeficientes de distribución de los elementos $A$ y $B\left(D_{\mathrm{A}} / D_{\mathrm{B}}\right)$.

$M e^{\mathrm{n}+}=$ Elemento metálico en general.

$\mathrm{Ln}^{3+}=$ Elemento lantánido.

$T R=$ Tierras raras.

$\mathrm{pH}_{50}=$ Valor del $\mathrm{pH}$ de la fase acuosa equilibrada en el que se extrae el $50 \%$ de la concentración del metal presente en la fase acuosa inicial.

\section{REFERENCIAS}

(1) Haskin, L.A. y PASTer, T.P. Handbook on the Physics and Chemistry of Rare Earths. Vol. 3. Eds. K.A. Gschneindner, Jr. y L. Eyring. North-Holland. Amsterdam, 1979: 1-80.

(2) Bautista, R.G. y WonG, M.M. Rare Earths, Extraction, Preparation and Applications. TMS. Warrendale (EE.UU.), 1989.

(3) Gupta, C.K. y Krisnamurthy, N. Int. Mater. Rev., 37 (5), 1992: 197-248.

(4) Bautista, R.G. Mineral Proc. Extractive Met. Rev., 8, 1992: 175-182.

(5) BAUTISTA, R.G. y JACKSON, N. Rare Earths, Resources, Science, Technology and Applications. TMS. Warrendale (EE.UU.), 1992.

(6) Habashi, F. Rare Earths'90. Proc. Int. Symp. on Processing of Rare Metals. Osaka (Japan), 1990: 47-52.

(7) Gschneinder, Jr., K.A. Handbook on the Physics and Chemistry of Rare Earths. Cumulative Index Vol. 1-15. Eds. K.A. Gschneinder, Jr. y L. Eyring. North-Holland. Amsterdam, 1993: 509-521.

(8) Donohue, T. J. Chem. Phys., 67 (11), 1977: 5.402-5.404.

(9) Qiu, L.F., Kang, X.H. y Wang, T.S. Sep. Sci. \& Technol., 26 (2), 1991: 199-221.

(10) HuBicki, Z. Hydrometallurgy, 23, 1990: 319-331.

(11) Gupta, C.K. y MukherJee, T.K. Hydrometallurgy in Extraction Processes. Vol. II. CRC Press. Boca Raton, 1990: 105-110. 
(12) Yanagihara, N., Vemulapalli, K., Fernando, Q. y DYKE, J.T. J. Less-Common Met., 167, 1991: 223-232.

(13) Fernando, Q., Yanagihara, N., Dyke, J.T. y VemulaPALLI, K. Patente EE.UU. 5045289. 1991.

(14) Ritcey, G.M. y AshbrooK, A.W. Solvent Extraction. Part II, Ed. Elsevier. Amsterdam, 1979: 316-420.

(15) AMER, S. Rev. Metal. CENIM, 17 (4), 1981: 245-283.

(16) Sherrington, L. Handbook of Solvent Extraction. Eds. T.C. Lo, M.H.I. Baird y C. Hanson. John Wiley \& Sons. Nueva York, 1983: 717-723.

(17) Preston, J.S. Hydrometallurgy, 14, 1985: 171-188.

(18) Du Preez, A.C. y Preston, J.S. Solv. Extr. Ion Exch., 10 (2), 1992: 207-230.

(19) SATo, T. Hydrometallurgy, 22, 1989: 121-140.

(20) Preston, J.S., Cole, M.P., DU Preez, A.C., Fox, M.H. y FLEMING, A.M. Hydrometallurgy, 41, 1996: 21-44.

(21) AMER, S. Rev. Metal. CENIM, 16 (6), 1980: 349-366.

(22) Ritcey, G.M. y AshbrooK, A.W. Solvent Extraction. Part I. Ed. Elsevier. Amsterdam, 1984: 87-171.

(23) Alguacil, F.J. Rev. Metal. CENIM, 23 (3), 1987: 172188.

(24) Urbanski, T.S., Abbruzzese, C., Fornari, P. y MassidDA, R. Hydrometallurgy, 28, 1992: 1-12.

(25) AbBruZzese, C., Fornari, P., Massidda, R. y URBANSKI, T.S. Hydrometallurgy, 28, 1992: 179-190.

(26) Urbanski, T.S., AbbruZzese, C., Fornari, P. y MassidDA, R. Hydrometallurgy, 25, 1990: 185-195.

(27) Urbanski, T.S., Fornari, P. y AbbruZzese, C. Hydrometallurgy, 40, 1996: 169-179.

(28) Hsu, K.H., Huang, C.H., King, T.C. y Li, P.K. Proc. ISEC'80. Vol. 2. Lieja (Bélgica), 1980: 80-82.

(29) Cerna, M., Volaufova, E. y Rod, V. Hydrometallurgy, 28, 1992: 339-352.
(30) Preston, J.S. Hydrometallurgy, 42, 1996: 151-167.

(31) Preston, J.S., Cole, P.M., Craig, W.M. y Feather, A.M. Hydrometallurgy, 41, 1996: 1-19.

(32) Huang, C.H., Hu, R.F. y Xu, G.X. Solv. Extr. Ion Exch., 7 (3), 1989: 489-497.

(33) Tang, J. y Wai, C.M. Anal. Chem., 58, 1986: 3.2333.235 .

(34) Manchanda, V.K., Chang, C.A. y Peng, J. Solv. Extr. Ion Exch., 6 (5), 1988: 835-837.

(35) Ensor, D.D., Nicks, M. y PRUett, D.J. Sep. Sci. \& Technol., 23 (12-13), 1988: 1.345-1.353.

(36) Ludwig, R., Inoue, K. y Yamato, T. Solv. Extr. Ion Exch., 11 (2), 1993: 311-330.

(37) Huang, C.H. y Bautista, R.G. Sep. Sci. \& Technol., 18, 1983: 1.667-1.683.

(38) Kondo, K., NaKashio, F. y Ohtsubo, H. Solv. Extr. Ion Exch., 10 (4), 1992: 655-662.

(39) Majdan, M., y Kolarik, Z. Solv. Extr. Ion Exch., 11 (2), 1993: 331-348.

(40) Kubota, F., Goto, M. y Nakashio, F. Solv. Extr. Ion Exch., 11 (3), 1993: 437-453.

(41) Freiser, H. Solv. Extr. Ion Exch., 6 (6), 1988: $1.093-$ 1.108.

(42) Zielinski, S., BuCA, M. y Szczepanik, A. Hydrometallurgy, 26, 1991: 243-254.

(43) Zielinski, S. y Szczepanik, A. Hydrometallurgy, 33, 1993: 219-226.

(44) Cox, M. Principles and Practices of Solvent Extraction. Eds. J. Rydberg, C. Musikas y G.R. Choppin. Marcel Dekker. Nueva York, 1992: 357-412.

(45) Zhang, B.Z., Lu, K.Y., King, K.C., WeI, W.C. y Wang, W.C. Hydrometallurgy, 9, 1982: 205-210. 